\title{
Aromatase inhibitors as adjuvant therapy: how will results of the ATAC trial at San Antonio 2004 and BIG 1-98 at St Gallen 2005 impact on aromatase inhibitors as adjuvant endocrine therapy?
}

\author{
N. S. Wong, K. I. Pritchard \\ Toronto Sunnybrook Regional Cancer Centre, University of Toronto, Toronto, Ontario, Canada.
}

\section{Introduction}

Despite declining mortality and a stabilization of annual incidence rates, breast cancer remains the commonest malignancy among North American women $[1,2]$.

Approximately two thirds of breast cancers are hormone sensitive, and 5 years of adjuvant therapy with the anti-oestrogen tamoxifen reduces breast cancer relapse by $41 \%$ and mortality by $34 \%$ in patients with hormone receptor positive early breast cancer [3].

Such success rates attained with adjuvant tamoxifen are tempered by disease relapse in a significant proportion of patients, the risk of which is highest in the first 2 years and continues for an indefinite period [4,5]. In addition, treatment is associated with a small but well-defined risk of serious complications including endometrial malignancies and thromboembolic disease. These limitations have been the impetus behind the search for new adjuvant endocrine treatments.

Third generation aromatase inhibitors prevent conversion of androgens to oestrogen in peripheral tissues, resulting in profound oestrogen deprivation in postmenopausal women.

These agents have shown at least equivalent or superior efficacy with a more favourable toxicity profile in the first line metastatic setting compared to tamoxifen, paving the way for trials testing their efficacy as

Correspondence to: Dr Kathleen I. Pritchard, Department of Clinical Trials and Epidemiology, Toronto Sunnybrook Regional Cancer Centre, 2075 Bayview Avenue, Toronto M4N 3M5, Ontario, Canada. E-mail: kathy. pritchard@sw.ca; Tel: +14164804616

Received 12/08/05

Accepted 12/08/05

BCO/395/2005/FO adjuvant therapy [6-10]. Over the past 3 years, the results of these high-quality large randomized controlled trials have been presented or published, leading to general acceptance of the aromatase inhibitors as important components of adjuvant endocrine therapy for postmenopausal women with primary breast cancer [11-21].

\section{Overview of aromatase inhibitors as adjuvant therapy}

\section{Arimidex, Tamoxifen Alone or in Combination}

The first published trial involving a third generation aromatase inhibitor as adjuvant therapy for breast cancer was Arimidex, Tamoxifen Alone or in Combination (ATAC), one of the largest adjuvant trial ever conducted [11]. This multinational double blind study enrolled 9366 postmenopausal women with invasive operable breast cancer who had completed primary therapy and were eligible to receive adjuvant hormonal therapy. Patients were randomized to receive anastrozole alone, tamoxifen alone or a combination of the two drugs for 5 years.

Patients in the study were well balanced for baseline characteristics and had a relatively good prognosis, with $61 \%$ having node-negative disease and $64 \%$ having $T 1 \mathrm{c}$ or smaller tumours. Eighty four percent of the patients had hormone receptor-positive breast cancer.

With a median follow-up of 33.3 months, anastrozole was found to be superior to tamoxifen in terms of disease-free survival, time to recurrence, and incidence of contralateral breast cancer (see Table 1). Insufficient events had occurred for formal analyses of distant disease free survival and overall survival at that time. 
Table 1. Key efficacy endpoints in the third generation aromatase inhibitor adjuvant studies.

\begin{tabular}{|c|c|c|c|c|}
\hline $\begin{array}{l}\text { Study/median } \\
\text { follow-up }\end{array}$ & $\begin{array}{l}\text { HR disease-free } \\
\text { survival }(95 \% \mathrm{Cl})\end{array}$ & $\begin{array}{l}\text { HR distant disease-free } \\
\text { survival }\end{array}$ & HR overall survival & $\begin{array}{l}\text { Contralateral breast } \\
\text { cancer }\end{array}$ \\
\hline \multicolumn{5}{|l|}{ ATAC } \\
\hline \multicolumn{5}{|l|}{33 months } \\
\hline ITT & $\begin{array}{l}0.83(0.71-0.96) \\
P=0.013\end{array}$ & - & - & $\begin{array}{l}\text { OR } 0.42(0.22-0.79) \\
P=0.007\end{array}$ \\
\hline Horm recep+ & $\begin{array}{l}0.78(0.65-0.93) \\
P=0.005\end{array}$ & - & - & $\begin{array}{l}\text { OR } 0.29(0.13-0.64) \\
P=0.002\end{array}$ \\
\hline \multicolumn{5}{|l|}{47 months } \\
\hline ITT & $\begin{array}{l}0.86(0.76-0.99) \\
P=0.03\end{array}$ & - & - & $\begin{array}{l}\text { OR } 0.62(0.38-1.02) \\
P=0.062\end{array}$ \\
\hline Horm recep+ & $\begin{array}{l}0.82(0.70-0.96) \\
P=0.015\end{array}$ & - & - & $\begin{array}{l}\text { OR } 0.56(0.32-0.98) \\
P=0.042\end{array}$ \\
\hline \multicolumn{5}{|l|}{68 months } \\
\hline ITT & $\begin{array}{l}0.87(0.78-0.97) \\
P=0.01\end{array}$ & $\begin{array}{l}0.86(0.74-0.99) \\
P=0.04\end{array}$ & $\begin{array}{l}0.97(0.83-1.14) \\
P=0.7\end{array}$ & $\begin{array}{l}\text { OR } 0.58(0.38-0.88) \\
P=0.01\end{array}$ \\
\hline Horm recep+ & $\begin{array}{l}0.83(0.73-0.94) \\
P=0.005\end{array}$ & $\begin{array}{l}0.84(0.70-1.00) \\
P=0.06\end{array}$ & $\begin{array}{l}0.97(0.85-1.12) \\
P=0.7\end{array}$ & $\begin{array}{l}\text { OR } 0.47(0.29-0.75) \\
P=0.001\end{array}$ \\
\hline \multicolumn{5}{|l|}{ MA17 } \\
\hline $\begin{array}{l}29 \text { months } \\
\text { ITT }\end{array}$ & $\begin{array}{l}0.57(0.43-0.75) \\
P=0.00008\end{array}$ & - & $0.76(0.48-1.21)$ & $46 \%$ reduction \\
\hline \multicolumn{5}{|l|}{30 months } \\
\hline ITT & $42 \%$ risk reduction & $40 \%$ risk reduction & - & $37.5 \%$ reduction \\
\hline Node-negative & $0.45(0.27-0.75)^{\star}$ & $0.63(0.31-1.27)$ & $1.52(0.76-3.06)$ & - \\
\hline Node-positive & $0.61(0.45-0.84)^{*}$ & $0.53(0.36-0.78)^{*}$ & $0.61(0.38-0.98)^{*}$ & - \\
\hline \multicolumn{5}{|l|}{ IES } \\
\hline 30.6 months & $\begin{array}{l}0.68(0.56-0.82) \\
P<0.01\end{array}$ & $\begin{array}{l}0.66(0.52-0.83) \\
P=0.0004\end{array}$ & $\begin{array}{l}0.88(0.67-1.16) \\
P=0.37\end{array}$ & $\begin{array}{l}0.44(0.20-0.98) \\
P=0.04\end{array}$ \\
\hline 37.4 months & $\begin{array}{l}0.73(-) \\
P=0.0001\end{array}$ & - & $\begin{array}{l}0.83(0.67-1.02) \\
P=0.08\end{array}$ & $\begin{array}{l}0.50(0.26-0.97) \\
P=0.04\end{array}$ \\
\hline \multicolumn{5}{|l|}{ ARNO 95/ABCSG } \\
\hline 28 months & $\begin{array}{l}0.60(0.44-0.81) \\
P=0.0009\end{array}$ & $\begin{array}{l}0.61(0.42-0.87) \\
P=0.0067\end{array}$ & $\begin{array}{l}0.76(0.52-1.12) \\
P=0.16\end{array}$ & - \\
\hline \multicolumn{5}{|l|}{ BIG 1-98 } \\
\hline 25.8 months & $\begin{array}{l}0.81(0.70-0.90) \\
P=0.003\end{array}$ & $\begin{array}{l}0.73 \\
P=0.0012\end{array}$ & $\begin{array}{l}0.86 \\
P=0.16\end{array}$ & $\bar{P}=0.125$ \\
\hline
\end{tabular}

-: not available. *Statistically significant. HR: hazard ratio; Horm recep+: hormone receptor positive; ITT: intention to treat; OR: odds ratio.

As expected, the disease-free survival and time to recurrence benefits with anastrozole were not seen in the hormone receptor-negative patient population. In addition, no benefit was observed in any of the stated end-points with combination therapy, leading to early closure of this study arm.

In terms of side-effects, anastrozole was significantly better tolerated than tamoxifen with respect to endometrial cancer $(P=0.02)$, vaginal bleeding and discharge $(P<0.0001$ for both), cerebrovascular events $(P=0.0006)$, venous thromboembolic events $(P=0.0006)$, and hot flushes $(P<0.0001)$. However, more patients on anastrozole compared to tamoxifen developed musculoskeletal disorders and fractures ( $P<0.0001$ for both).

The safety and efficacy results of ATAC were updated at a median follow-up of 47 months [12].
Key efficacy end-points at 4 years remained significantly more favourable with anastrozole compared to tamoxifen (see Table 1) and the updated safety analysis also confirmed the findings of the first analysis. Incidence rates of bone fractures, while higher in the anastrozole arm, remained constant over time.

Results of the completed treatment analysis presented at San Antonio Breast Cancer Symposium (SABCS) 2004 will be discussed below.

\section{MA-17}

The second study involving a third generation aromatase inhibitor employed a different strategy. The National Cancer Institute of Canada Clinical Trials Group (NCIC CTG) MA-17 trial was a multicentre, 
double-blind, placebo-controlled trial testing the effectiveness of 5 years of letrozole therapy vs. placebo in postmenopausal women with breast cancer who had completed 5 years of tamoxifen therapy [15].

A total of 5187 women were enrolled. Fifty percent of patients had node-negative breast cancer and $98 \%$ were hormone receptor-positive.

With a median follow-up of 2.4 years, disease-free survival was significantly higher among patients receiving letrozole compared to placebo. There was no observed difference in overall survival $(P=0.25)$ at the initial analysis.

Low-grade hot flashes, arthritis, arthralgia and myalgia were more frequent in the letrozole group, but vaginal bleeding was less frequent. There was a trend towards increased new diagnoses of osteoporosis in women in the letrozole group compared to women in the placebo group $(P=0.07)$ but fracture rates were similar.

Given these positive findings after the first interim analysis, the independent data and safety monitoring committee recommended unblinding of the results to the participants. Those participants receiving placebo were offered letrozole and about $70 \%$ have accepted (Goss P, personal communication).

The updated results of MA-17 were presented at the 2004 American Society of Medical Oncology Annual Meeting (see Table 1). In subgroup analysis, letrozole improved disease-free survival in both node-negative patients and node-positive patients. Significant prolongation of distant disease-free survival and overall survival was observed in nodepositive but not in node-negative patients [16].

Safety data remained essentially similar to the first analysis. There was no increase in the incidence of cardiac events, hypercholesterolemia or treatment discontinuation due to adverse events in the letrozole arm as compared to placebo. Although there was a significantly higher rate of patient reported new osteoporosis $(P=0.003)$ with letrozole, fracture rates were similar.

\section{Intergroup Exemestane Study}

In the Intergroup Exemestane Study (IES), 4742 postmenopausal women with hormone receptor positive or unknown breast cancer who remained disease free after completing 2-3 years of adjuvant tamoxifen were randomized to exemestane or continued therapy with tamoxifen for a total duration of 5 years of endocrine therapy in a double blind, multicentre Phase III trial [17]. Approximately $50 \%$ of patients were node-negative and oestrogen-receptor status was unknown in $17 \%$ of patients.

With a median follow-up of 30.6 months, exemestane significantly reduced the risk of first events by
$32 \%$ and contralateral breast cancer by $56 \%$ compared to tamoxifen. The absolute benefit in diseasefree survival was $4.7 \%(95 \% \mathrm{Cl}, 2.6-6.8)$ at 3 years. Distant disease-free survival was also better in the exemestane group although no survival benefit was observed. Exemestane was equally effective regardless of progesterone receptor or nodal-status.

Treatment discontinuation as a result of adverse events occurred in $5.8 \%$ of patients receiving exemestane compared to $5.0 \%$ of patients receiving tamoxifen ( $P$-value not given). Patients treated with exemestane had a higher incidence of arthralgia, osteoporosis and fractures. Conversely, vaginal bleeding, cramps, thromboembolic events and endometrial cancer were more frequent in the group which received tamoxifen.

Updated data from the IES study with a median follow-up of 37.4 months has been presented [18]. Exemestane remained significantly superior to tamoxifen in terms of disease-free survival, breast-cancerfree survival, and time to second breast cancer. A trend towards improved overall survival among the exemestane patients did not reach statistical significance $(P=0.08)$. Updated adverse event data has also been presented, with a specified $P$-value of less than 0.01 taken as the level of significance due to the multiple analyses carried out. More patients in the exemestane arm (20 vs. $8, P=0.01)$ developed myocardial infarction, although this difference did not meet the specified level of statistical significance. Cardiac mortality was similar in the two groups.

\section{Arimidex Nolvadex 95/Austrian Breast Cancer Study Group Trial 8}

The fourth study of adjuvant aromatase inhibitor therapy is a combined analysis of the Arimidex Nolvadex (ARNO 95) Trial and the Austrian Breast Cancer Study Group (ABCSG) Trial 8, presented at the 2004 SABCS [19].

These trials assessed the merit of switching to anastrozole after 2 years of tamoxifen compared to continuing tamoxifen treatment for the remaining 3 years of adjuvant therapy. Both studies had similar designs and broadly similar inclusion criteria and were prospectively planned for combined analysis.

A total of 3123 patients were included in the two trials. Mean age was 63 years (range $41-80$ ), $74 \%$ of patients were node-negative, $70 \%$ had $\mathrm{T} 1$ tumours and all patients were hormone receptor-positive and chemo-naive.

After a median follow-up of 28 months, the estimated 3-year event-free survival was $95.8 \%$ in the anastrozole arm and $92.7 \%$ in the tamoxifen arm (hazard ratio $(\mathrm{HR}): 0.60 ; 95 \%$ confidence interval $(\mathrm{Cl})$ : $0.44-0.81 ; P=0.0009$ ), mainly as a result of 
improved distant disease-free survival (HR: 0.61; 95\% Cl: $0.42-0.87 ; P=0.0067)$. The benefit of switching to anastrozole was seen irrespective of nodal-status and progesterone-receptor status but was not evident in patients with Grade 3 tumours.

Treatment with anastrozole was generally well tolerated. However, more patients treated with anastrozole developed fractures compared to patients randomized to tamoxifen.

\section{Italian Tamoxifen Anastrozole}

The Italian Tamoxifen Anastrozole (ITA) Study randomized 448 women who were recurrence free after 2 years of tamoxifen to receive either anastrozole or tamoxifen for a total duration of 5 years [20]. With a median follow-up of 36 months, disease free (HR: 0.35 ; $95 \% \mathrm{Cl}: 0.18-0.68 ; P=0.001$ ) and local recurrence-free survival (HR: 0.15; 95\% Cl: 0.03-0.065; $P=0.003$ ) were significantly improved in the anastrozole group. No survival data has been reported as yet.

\section{ATAC at SABCS 2004}

The ATAC completed treatment analysis was presented at the 2004 SABCS and included the first mature analysis of distant relapse free and overall survival $[13,14]$. After a median follow-up of 68 months, only $8 \%$ of patients remained on active therapy.

Anastrozole compared to tamoxifen significantly improved disease-free survival (HR: 0.87; $P=0.01)$ and time to recurrence (HR: $0.79 ; P=0.00050)$ and reduced contralateral breast cancer (HR: 0.58; $P=$ 0.01 ) in the intention-to-treat population (see Table 1). The benefits in disease-free survival (HR: 0.83; $P=$ 0.005), time to recurrence (HR: 0.74; $P=0.0002$ ) and reduction in contralateral breast cancer (HR: $0.47 ; P=0.001$ ) were greater in the hormone receptor positive subgroup. The absolute differences in disease-free survival and time to recurrence were $3.3 \%$ and $3.7 \%$, respectively.

There was a trend towards improved time to distant recurrence (HR: 0.84; 95\% Cl: 0.70-1.00; $P=$ $0.06)$ among hormone receptor-positive patients. Time to breast cancer death (HR: $0.87 ; P=0.2$ ) and overall survival (HR: $0.97 ; P=0.7)$ were not significantly different in the two arms.

The benefit of anastrozole was seen regardless of nodal-status, tumour size and prior exposure to chemotherapy.

Safety and tolerability data previously demonstrated by anastrozole were maintained with longer follow-up. No new safety concerns were identified. Thromboembolism, vaginal bleeding, endometrial cancer, ischaemic cerebrovascular events and hot flashes were significantly less common with anastrozole compared to tamoxifen while fracture rates were significantly higher with anastrozole. There was no significant difference in the rate of ischaemic cardiovascular disease. Withdrawals due to adverse events were significantly lower in the anastrozole group $(11.1 \%$ vs. $14.3 \%, P=0.0002)$, as were drug related serious adverse events $(4.7 \%$ vs. $9.0 \%$, $P<0.0001)$.

\section{Breast International Group 1-98 at St Gallen}

While we know from published trials that the use of an adjuvant aromatase inhibitor improves breast cancer outcomes when given in place of, or after 2 , 3 or 5 years of tamoxifen, there is ongoing controversy regarding the optimal aromatase inhibitor and the best sequence of administration.

The Breast International Group (BIG )/International Breast Cancer Study Group (IBCSG) 1-98/Femara Tamoxifen (FEMTA) trial will seek to answer some of these important questions. This study completed accrual in May 2003 and preliminary results were presented at the 9th International Conference on Primary Therapy of Early Breast Cancer held in St Gallen, Switzerland [22].

This multicentre double blind study randomized 8010 postmenopausal women with endocrine-responsive early-stage breast cancer to one of four arms: tamoxifen for 5 years, letrozole for 5 years, tamoxifen for 2 years followed by letrozole for 3 years or the reverse sequence of letrozole for 2 years followed by tamoxifen for 3 years. The primary core analysis included all patients, excluding events and followup beyond 2 years for patients in the latter two arms involving a switch in endocrine therapy. Fifty-two percent of patients had node-negative breast cancer and approximately $37 \%$ had tumours greater than $2 \mathrm{~cm}$. Twenty five percent of patients had prior chemotherapy. Four thousand and three patients were treated initially with letrozole and 4007 with tamoxifen.

With a median follow-up of 25.8 months, the projected 5 year disease-free survival was $84 \%$ with letrozole and $81.4 \%$ with tamoxifen (absolute difference 3.4\%, HR: 0.81; 95\% Cl: 0.70-0.93; $P=0.003$ ), mainly due to reduction of distant metastases (HR: $0.73 ; P=0.0012$ ) in favour of letrozole. However, no significant difference in overall survival has been detected to date (HR: 0.86; $P=0.16$ ). In subgroup analysis, letrozole reduced the risk of recurrence by $29 \%$ in patients with node-positive breast cancer and by $30 \%$ in patients who had received prior chemotherapy.

In terms of safety, hypercholesterolemia $(43.5 \%$ vs. $19.2 \%$, more than $80 \%$ of cases were Grade 1 ) and bone fractures $(5.8 \%$ vs. $4.1 \%)$ were significantly 
Table 2. BIG 1-98/FEMTA adverse events.

\begin{tabular}{lcc}
\hline & Letrozole (\%) & Tamoxifen (\%) \\
\hline Hot flushes & 33.6 & 38.1 \\
Night sweats & 14.0 & 16.2 \\
Nausea & 8.8 & 9.5 \\
Vomiting & 2.7 & 2.6 \\
Vaginal bleeding & 3.3 & 6.6 \\
Endometrial biopsies & 1.9 & 7.2 \\
Invasive endometrial cancer & 0.2 & 0.4 \\
Hypercholesterolemia* & 43.6 & 19.2 \\
Thromboembolism & 1.0 & 2.4 \\
CVA/TIA & 1.2 & 1.1 \\
Cardiovascular events & 8.7 & 8.3 \\
Bone fracture & 5.8 & 4.1 \\
\hline
\end{tabular}

*>80\% of hypercholesterolemia was Grade 1 (Grade 1 events: LET 35.1\%/TAM 17.3\%), based on non-fasting levels/single measurements.

more common with letrozole (see Table 2). There was a trend towards a lower incidence of invasive endometrial cancer with letrozole $(0.2 \%$ vs. $0.4 \%$; odds ratio (OR): 0.40$)$, but this did not reach statistical significance $(P=0.078)$.

Updated safety data presented at the 2005 American Society of Clinical Oncology Annual Meeting [23] showed fewer total and cancer-specific deaths among patients who received letrozole but a trend towards higher 5-year incidence of death without recurrence on the letrozole arm that was not statistically significant $(3.1 \%$ vs. $1.8 \% ; P=0.08)$. This was mainly a result of more observed cerebrovascular accident deaths (7 vs. 1 ) and cardiac deaths (13 vs. 6) with letrozole.

\section{Interpretation and implications}

Both the ATAC completed treatment analysis and the BIG 1-98/FEMTA primary core analysis provide further evidence that the aromatase inhibitors as a class can improve breast cancer outcomes. Now either anastrozole or letrozole can be considered as front line therapeutic options in place of tamoxifen after completion of primary therapy for hormone receptor-positive breast cancer in postmenopausal women. Both drugs have shown improvements in disease-free survival and distant relapse-free survival. In addition a significant reduction in contralateral breast cancer was observed in ATAC and a similar trend was observed in BIG 1-98. Questions regarding the optimal timing and sequence of therapy can only be answered when results are available from the cross-over study in BIG 1-98.

The safety advantages of the aromatase inhibitors are highlighted in these two trials, including fewer thromboembolic complications and endometrial cancers relative to tamoxifen. Conversely bone fractures are more common with the aromatase inhibitors compared to tamoxifen and monitoring of bone mineral density is important in patients who receive long-term treatment with these agents.

There were more cardiovascular deaths observed among patients randomized to letrozole in BIG 1-98. These observations are mirrored in the follow-up safety data from the IES study, which also revealed a higher number of cardiovascular events that did not reach statistical significance. As both studies compared an aromatase inhibitor with tamoxifen, it is not possible to determine if these observed differences in cardiac events resulted from a detrimental effect of the former agent or a protective effect of the latter drug. Of note, MA-17 is the only adjuvant study with a design comparing an aromatase inhibitor with placebo. In this study, there was no increase in cardiovascular events with letrozole treatment, suggesting that aromatase inhibitors may not have an adverse effect on cardiovascular events per se.

Overall, preliminary results from BIG 1-98 and the ATAC completed treatment data provide exciting new insights that contribute further to patient care and highlight the need for careful monitoring of potential treatment related complications.

\section{References}

1. National Cancer Institute Canada. Canadian Cancer Statistics 2004. http://www.ncic.cancer.ca

2. American Cancer Society. Cancer Facts and Figures 2004. http://www.cancer.org

3. Early Breast Cancer Trialists' Collaborative Group (EBCTCG). Effects of chemotherapy and hormonal therapy for early breast cancer on recurrence and 15-year survival: an overview of the randomized trials. Lancet 2005; 365: 1687-1717.

4. Early Breast Cancer Trialists' Collaborative Group. Systemic treatment of early breast cancer by hormonal, cytotoxic, or immunotherapy: 133 randomised trials involving 31000 recurrences and 24000 deaths among 75000 women. Lancet 1992; 339: 71-85.

5. ATAC Trialists' Group Writing Committee. ATAC trial update: author reply. Lancet 2005; 365: 1225-1226.

6. Paridaens R, Therasse P, Dirix L, et al. First line hormonal treatment $(\mathrm{HT})$ for metastatic breast cancer (MBC) with exemestane (E) or tamoxifen (T) in postmenopausal patients (pts) - A randomized phase III trial of the EORTC Breast Group. Proc ASCO 2004; 22(14S) [Abstract 515].

7. Nabholtz JM, Buzdar A, Pollak M, et al. Anastrozole is superior to tamoxifen as first-line therapy for advanced breast cancer in postmenopausal women: results of a North American multicenter randomized trial. Arimidex Study Group. J Clin Oncol 2000; 18: 3758-3767.

8. Bonneterre J, Thurlimann B, Robertson JF, et al. Anastrozole versus tamoxifen as first-line therapy for advanced breast cancer in 668 postmenopausal women: results of the Tamoxifen or Arimidex Randomized Group Efficacy and Tolerability study. J Clin Oncol 2000; 18: 3748-3757. 
9. Bonneterre J, Buzdar A, Nabholtz JM, et al. Anastrozole is superior to tamoxifen as first-line therapy in hormone receptor positive advanced breast carcinoma. Cancer 2001; 92(9): 2247-2258.

10. Mouridsen $H$, Gershanovich $M$, Sun $Y$, et al. Phase III study of letrozole versus tamoxifen as first-line therapy of advanced breast cancer in postmenopausal women: analysis of survival and update of efficacy from the International Letrozole Breast Cancer Group. J Clin Oncol 2003; 21: 2101-2109.

11. The ATAC (Arimidex, Tamoxifen Alone or in Combination) Trialists' Group. Anastrozole alone or in combination with tamoxifen versus tamoxifen alone for adjuvant therapy of postmenopausal women with early-stage breast cancer: first results of the ATAC randomized trial. Lancet 2002; 356: 2131-2139.

12. Baum M, Buzdar AU, Cuzick J, et al. Anastrozole alone or in combination with tamoxifen versus tamoxifen alone for adjuvant therapy of postmenopausal women with early-stage breast cancer: Results of the ATAC (Arimidex, Tamoxifen Alone or in Combination) trial efficacy and safety update analysis. Cancer 2003; 98: 1802-1810.

13. Howell A. The ATAC (Arimidex, Tamoxifen Alone or in Combination) trial in postmenopausal women with early breast cancer-updated efficacy results based on a median follow-up of 5 years. Breast Cancer Res Treat 2004; 88(Suppl 1): S7 [Abstract 1].

14. Howell A, Cuzick J, Baum M. Results of the ATAC (Arimidex, Tamoxifen, Alone or in Combination) trial after completion of 5 years' adjuvant treatment for breast cancer. Lancet 2005; 365: 60-62.

15. Goss PE, Ingle JN, Martino S, et al. Randomized trial of letrozole following tamoxifen as extended adjuvant therapy in receptor-positive breast cancer: updated findings from NCIC CTG MA.17. J Natl Cancer Inst 2005; 97(17): 1262-1271.

16. Goss PE, Ingle JN, Martino S, et al. Updated analysis of the NCIC CTG MA.17 randomized placebo $(P)$ controlled trial of letrozole $(\mathrm{L})$ after five years of tamoxifen in postmenopausal women with early stage breast cancer. Proc ASCO 2004; 22(14S) [Abstract 847].

17. Coombes RC, Hall E, Gibson LJ, et al. A randomised trial of exemestane after two or three years of tamoxifen therapy in postmenopausal women with primary breast cancer. N Engl J Med 2004; 350: 1081-1092.

18. Coombes RC, Hall E, Snowdon CF, Bliss JM. The Intergroup Exemestane Study: a randomized trial in postmenopausal patients with early breast cancer who remain disease-free after two to three years of tamoxifen-updated survival analysis. Breast Cancer Res Treat 2004; 88(Suppl 1): S7 [Abstract 3].

19. Jakesz R, Kaufmann M, Gnant M, et al. Benefits of switching postmenopausal women with hormone-sensitive early breast cancer to anastrozole after 2 years adjuvant tamoxifen: combined results from 3,123 women enrolled in the ABCSG Trial 8 and the ARNO 95 Trial. Breast Cancer Res Treat 2004; 88(Suppl 1): S7 [Abstract 2].

20. Boccardo F, Rubagotti A, Puntoni M, et al. Switching to anastrozole versus continued tamoxifen treatment of early breast cancer: preliminary results of the Italian Tamoxifen Anastrozole Trial. J Clin Oncol 2005; 23(22): 5138-5147 (e-pub 11 July 2005).

21. Winer EP, Hudis C, Burstein HJ, et al. American Society of Clinical Oncology Technology assessment on the use of aromatase inhibitors as adjuvant therapy for postmenopausal women with hormone receptor-positive breast cancer: status report 2004. J Clin Oncol 2005; 23: 619-629.

22. BIG 1-98 Collaborative Group. S4 Letrozole vs. Tamoxifen as adjuvant endocrine therapy for postmenopausal women in receptor-positive breast cancer. BIG 1-98: A prospective randomized double-blind phase III study. The Breast 2005; 14(Suppl 1): S3.

23. Thurlimann BJ, Keshaviah A, Mouridsen H, et al. BIG 1-98: Randomized double blind phase III study to evaluate letrozole (L) vs. tamoxifen ( $\mathrm{T}$ ) as adjuvant endocrine therapy for postmenopausal women with receptor-positive breast cancer. Proc ASCO 2005; 23: 6S [Abstract 511]. 